\title{
Management Education
}

An International Journal

How to Make Value Out of Advanced

Technology Projects

Supporting Experience Sharing 
MANAGEMENT EDUCATION: AN INTERNATIONAL JOURNAL

www.organization-studies.com

First published in 2015 in Champaign, Illinois, USA

by Common Ground Publishing LLC

www.commongroundpublishing.com

ISSN: 2327-8005

(C) 2015 (individual papers), the author(s)

(C) 2015 (selection and editorial matter) Common Ground

All rights reserved. Apart from fair dealing for the purposes of study, research, criticism or review as permitted under the applicable copyright legislation, no part of this work may be reproduced by any process without written permission from the publisher. For permissions and other inquiries, please contact cgsupport@commongroundpublishing.com.

Management Education: An International Journal is

peer-reviewed, supported by rigorous processes of criterion-

referenced article ranking and qualitative commentary, ensuring

that only intellectual work of the greatest substance and highest significance is published. 


\title{
How to Make Value Out of Advanced Technology Projects: Supporting Experience Sharing
}

\author{
Johan Holmqvist, Luleå University of Technology, Sweden
}

\begin{abstract}
Actionable knowledge is a competitive advantage to most organizations today. It is difficult to manage knowledge in technical projects, however, because both creation and use are embedded in the daily work. Experiences need to be extracted from activities, reflected on, and then contextualized again if they are going to benefit another project. The purpose of this qualitative study is to investigate experience sharing in early product development work and from that contribute to improved capture and formalization of experiences. The importance of a continuous learning process is stressed, but so is the capability of directed knowledge conversations. A demonstrator supporting such activities has been developed and partly tested. Not only the outline of the demonstrator, called the knowledge compass, but also the rationale are presented in this paper. The guiding questions included in the demonstrator have been evaluated as a sound basis of experience sharing for company representatives.
\end{abstract}

Keywords: Knowledge Conversation, Knowledge Sharing, Knowledge Transfer, Sharing Experiences

\section{Introduction}

$\mathrm{K}$ nowledge has long been an important asset to most organizations, and the topic of knowledge transfer and/or sharing is stressed from various perspectives (Argote and 2000). Knowledge is often divided in the literature into two main conceptsnamely, tacit, which relates to subjective experiences, and explicit, which refers to known facts. To make this distinction, these concepts are outlined as two separate entities in the literature. Polyani (1966a) has elaborated on the difference between explicit and tacit knowledge, and Nonaka (1994) has further contributed to his work. Explicit knowledge is thus codified facts and is often described as articulated knowledge, whereas tacit knowledge is often expressed in terms of metaphors visualizing practical and action-oriented dimensions Nonaka (1994). Tacit knowledge (Polyani 1966a) also forms the structure that is needed for interpreting and making use of explicit knowledge. This is referred to as "pre-knowledge," "prior knowledge" or "pre-existing knowledge," and is applied by individuals as a coding key for building new knowledge when doing something new, i.e. - that is, the reflective processes of problem solving (Schön 1983). Drawing the descriptions towards the ends of a continuum is thus done to simplify the process. Polyani $(1966 \mathrm{~b}, 7)$ concludes on the dependency between the two types:

"All knowledge is either tacit or rooted in tacit knowledge. A wholly explicit knowledge is unthinkable."

Tacit and explicit knowledge are complementary, which makes it complex to actually transfer factual knowledge and to share experiences so that they benefit subsequent projects; the process is not straightforward. Today companies that act on a business-to-business market are simultaneously working in a global environment. The trends toward offering a 'total solution' and for taking responsibilities for the life-cycle perspective call for managing additional skills and competences. Moreover, the act of identifying and closing knowledge gaps - for example, white spots, or areas that are unknown or sparsely investigated - is related to the core competitive actions that need to be fulfilled to support development.

Knowledge gaps have been identified in literature, from a researcher and scientific community point of view competence mapping have highlighted that gaps that exists often depends on organisational members and personal relationship (Volpentesta and Felicetti, 2013). In this research the level of these knowledge gaps are addressed as: for example; individual level, project

Management Education: An International Journal

Volume 15, Issue 4, 2015, www.techandsoc.com, ISSN 2327-8005

(C) Common Ground, Johan Holmqvist, All Rights Reserved

Permissions: cg-support@commongroundpublishing.com 
level, technology area, or company level. Capitalizing on available knowledge assets and making the most out of them is crucial to execute a more successful project, especially during handovers, when companies are shifting from manufacturing to total solutions. A company representative in this study reflected on this knowledge shift:

"We have moved from a company selling products to providing a system of intertwined products and services. How do we capitalize on our knowledge assets when the value we offer is no longer found solely in the physical goods?" (Respondent 1)

The shift toward total solutions requires development teams to use, adapt, and reuse their dayto-day experiences in an innovative way, for instance, to meet sustainability demands and competition from new actors in upcoming markets: Brazil, Russia, India, and China (so-called BRIC countries). The purpose of this study is to investigate - framed by an assumed perspective shift in knowledge management - experience sharing in advanced engineering projects. The consequences of the sharing actions will be discussed in order to address a "closure" of knowledge gaps in technology areas.

\section{Methodology}

The study is based on empirical data from teams in a manufacturing industry. A literature study supported the work and started early on in the studies with a search of articles related to the initial research topics. The keywords that guided the search included knowledge management, knowledge sharing, knowledge transfer, knowledge conversation, experience sharing, product development, expertise, experiences, tacit knowledge, and explicit knowledge.

From the empirical study knowledge transfer between two subsequent types of projectsadvanced engineering and new product development - has provided a base for the data generation. In particular, knowledge transfer in and between project members has been of interest. The project members not only possess different skills, but are also from different parts of the organization (i.e., both hierarchically and geographically dispersed); therefore, factors that constitute situations that need alternative or complementary support have also been possible to explore.

Visits to the company's sites, primarily in Europe but also in the United States and Asia, have been made to investigate project activities to collect data and to better understand the social dimensions. Data were gathered from project meetings and from shadowing employees in their daily work. The employees were all part of the technology project organization involved in these knowledge transfer situations. The employees have different responsibilities and roles: project leaders, managers, experts, and engineers. Field notes were taken during observations. Regular check-ins on internal project platforms and systems are also part of the data that have been collected. Data have been gathered over a time period of three years. This has provided a rich base on which to contextualize data and to develop a thorough understanding of the company's overall knowledge management approaches.

More specifically, 17 interviews have been conducted from May 2010 to August 2013. The duration of the interviews varied, but most lasted 45-60 minutes. Respondents were company representative involved in two project types at the company. These interviews were done face-toface during visits but also were supported by distributed technology (e.g., conferencing software). Respondents were engineers and project managers from the both types of projects. One project type deals with technology investigations and conceptualization, here called a knowledge project, and one project type deals with industrialization and exploitation, here called an execution project. Transferring knowledge and experiences from one to another of these project types is therefore crucial for the continuum.

Respondents were encouraged to formulate and express their thoughts freely in relation to a number of topics. The topics were knowledge and knowledge transfer, experiences, reflections, and feedback in relation to the format established by the company. The respondents were "gently" 
brought back to a topic if they started to deviate too far from it, which is in line with the format of semi-structured interviews (Fontana and Frey 2000). The researcher can pose follow-up questions to probe in-depth the answers of the respondents, and this was also done in the interviews. Experiences or knowledge intentionally were not defined, but were only stated as a topic to talk about. This approach made it possible for the respondents to clarify and define their perspectives on the terms and how they use the support methods and format provided by the company. The interviews were all recorded and transcribed, and later a software program (NVivo) (QSR International 2012) was used to perform further text analysis on both interview data and 25 textbased reports (7-12 pages each) from the projects. Employees involved in the knowledge projects contributed to these reports (as individuals who possess and communicate the knowledge). Thus, respondents from execution projects were the main target group of such documents; they were considered individuals who should be able to interpret the knowledge and make use of it in the project. Both projects were acting within the established company processes. The software offers a search query, which made it possible to search for a word or a sequence of words to find in what context they appear. This provided a pointer for places where situation reflections were made and also for where they were recognized.

During site visits, presentations and workshops have been performed to let the company representative, first, express his or her challenges and, second, discuss alternative solutions and test a demonstrator for supporting experience sharing and reflecting upon the situation, giving feedback to the researcher, and discussing alternatives.

\section{Knowledge Activities from a Life-Cycle Perspective}

Knowledge-intensive activities - for example, product development-incorporate knowledge from sources within and outside the organization (Blessing and Wallace 1998). If knowledge is retrieved and adapted to a new context the "use of knowledge" is successful, as opposed to a situation where knowledge is retrieved and not useful in the new situation. Yet new knowledge is generated in both cases, which is part of the knowledge base that continuously needs to be maintained and updated. Developing knowledge support is challenging because of the variety of issues, the complexity in the relationship between these issues, and their dynamic nature. Blessing and Wallace $(1998,27)$ state: "The dynamic nature of knowledge is determined by the generation of new knowledge as a result of knowledge use, that is a continual learning process." Thus, the support should consider a knowledge life-cycle, not only the actual knowledge in use. Knowledge life-cycle views have been proposed by numerous researchers based on studies in different design projects (cf. Blessing and Wallace 1998; Rowley 2001; Nuzzo and Lockwood 2005). There are different models of knowledge life-cycles, and it should be mentioned that more than one life-cycle could be considered in a single case due to influences from external sources, but also that not all stages in a life-cycle may be applicable in all types of contexts.

One knowledge life-cycle (Nuzzo and Lockwood 2005) suggests a number of steps defined as: identify, capture, store, access, share, use, learn, and generate/acquire. This life-cycle has been adapted and used to analyse the data in this study. The focus is on four of the suggested stages:

- Identify - Includes the activity of identifying knowledge and making knowledge assets and sources visible to the organization.

- Organize - Addresses an explicit view of codifying and linking knowledge elements to other elements in a knowledge structure. Tacit knowledge is also organized; however it is highly contextual, and the link between different contexts may be difficult to obtain. 
- Share-An activity initiated by individuals. Refers to the act of making knowledge (in this case) available to others, a collaborative action that leads to individual learning and conversations. It may also contribute to organizational learning (Ipe 2003).

- Learn - a process to reflect upon performed actions and use of knowledge, to build up the knowledge base through continuous feedback loops.

\section{Identification and Linking of Knowledge Assets at the Company}

Knowledge is identified at the company and interpreted in various ways depending on its original context. The company unit where knowledge of the project belongs has described their mission to be:

\section{“...Identify and close knowledge gaps.” (Company documentation)}

This focus should also guide other parts of an organization, such as execution projects, which are considered to be receivers of results from knowledge projects. However, the purpose of the execution project has been described as delivering new product-based objectives from both business and product demands. This shows that the intended purposes of the two types of projects are not the same and should not be. Nevertheless, these projects often depend on each other's experiences. To address a knowledge gap in one project and thereafter link identifiable knowledge assets from similar contexts is a challenge, but also a learning situation of utmost concern.

Challenges that these teams experience are occasionally that a problem could not be understood without knowing its context; for instance, respondents have explained how difficult it is to use reports from the past if they have not been involved in such project before. Hence, engineers cannot fully understand the problem until they have developed a solution.

Knowledge conversations can support identifying knowledge assets as an activity, "linking" addressed knowledge gaps and knowledge assets from different contexts - adding one part to another and thereby gaining understanding of a potential knowledge gap, which will support the organization to prioritize knowledge activities and possibly close knowledge gaps.

By both observing project teams in their daily work and by analysing the documentation they produce from development activities, the team seems to (from an outsider's perspective) communicate messages in the contexts such as project, technology, product element, and relation to other aspect of the product, for example, time, place, activity, issue or task. Seldom is their message articulated as experience from a performed activity rather than communicating the result of it, in relation to the context listed above. This means that if one is not familiar with that specific project or technology investigation and preconditions are not described, the value of continuing a knowledge conversation is diminished. Addressing the situation by explaining something about the background and context is one way to enhance the value of these knowledge conversations.

Experiences from co-workers, communities of practice, the whole organization and what employees know themselves need to be linked with a context in which the experiences matters. Supporting individuals to communicate their knowledge assets and to see correlations to potential knowledge gaps is of utmost importance, and can benefit both individuals and organizations (Cheong and Tsui 2010). The organizational culture should stress the value of employees' knowledge; this should have an effect on what the individuals identify as organizational knowledge assets and thereby share with others.

The knowledge life-cycle stages of identify and organize focus on these issues. These stages are used as a reference point for what a knowledge conversation in general should incorporate at the beginning. Two themes of questions are here highlighted under describe and explain: 
- Describe

- ...what was the context of your matter?

○ ...what was the objective of your task?

- Explain

- ...what knowledge gap you addressed?

○ ...what was your experience?

○ ...your contribution to current practice?

\section{Supporting Knowledge Conversations and Shared Reflections}

Experiences from activities that were successfully—or unsuccessfully-completed should be seen as a learning opportunity. A respondent stated that experiences are about:

"Understanding consequences of past decisions and actions that were performed." (Respondent 3)

Therefore one can say that experiences derive from activities that they should support in ongoing as well as future projects (Disterer 2002). Purposeful knowledge conversations aside from technical problem-solving activities are rare, but are also important. One respondent explained that he likes to discuss in a team environment, even sharing personal experiences that may or may not relate to the project, or as he framed it:

"I think it is very important... because it is a learning experience I like to get everybody else's perspective. I think overall, we don't necessarily get to pick the people on our team all the time, so you have different personalities and different viewpoints." (Respondent 4)

This is interpreted as expressing or articulating experiences to others in order to make the process more tangible in reflecting on specific activities. In addition, the importance of knowledge conversations is stressed by the respondent, not only when experiences are directly related to the project itself, but also when the conversation involves different perspective of the situation in focus.

Recommending actions has been recognized as valuable support in development activities, both in on-going projects and in adapting to new contexts. Introducing questions in knowledge conversations supports the procedure for how experiences are shared and reflected upon. Two of the knowledge life-cycle stages addressed earlier in this paper-sharing and learning - inspired the following two themes of statements: to clarify and to orchestrate. To clarify and to encourage individuals to reflect on their actions and the consequences of these situations. To orchestrate aims at how to direct experiences to a role, which can then execute or bring experiences into action to achieve the desired effect. Statements to support such activities are:

- Clarify

- ...who will directly learn from your experience!

- ...how your experience can be carried into actions! 
- Orchestrate your experiences

○ ...to those that will best need them!

○ ...to specific roles, levels, resources etc.

○ ...to close other knowledge gaps!

\section{Knowledge Conversations}

Companies apply an approach to knowledge management that intends to support creation and sharing of knowledge as a routine in daily work and not as a specialized activity in itself. In such an environment, it is more of an attitude or a culture - where engineers, management and experts create and share knowledge - than a prescribed procedure (Nonaka 1991).

Knowledge worker is a term used in the literature (Davenport and Prusak 1998) to describe a person who works for a living with the tasks of developing and/or using knowledge. Preferably, knowledge workers possess both technical and socially constructed knowledge. However, knowledge workers have to not only manage and coordinate knowledge, but they also need to reflect upon results of their knowledge activities. Webber claims that:

"Conversations are the way knowledge workers discover what they know, share it with their colleagues, and in the process create new knowledge for the organization." (Webber 1993, 28)

This excerpt can be interpreted as stressing the importance of conversations and shared reflections in an organization. From one perspective, knowledge workers are everyone who uses knowledge in his or her work, not considering how it is used or by what purpose. The other perspective stresses creation, development, reflection, and sharing of knowledge. Product development includes different phases with defined activities. A generic product development process starts in a planning phase, identifying needs, setting up requirements, and clarifying problems. This phase is followed by technology development, which might lead to concept development, detailed design, and later making the transition into production.

Consequently, some individuals create and develop new knowledge and others are focused on using that knowledge as part of their profession. Even though technology development, creation, and further development of knowledge often relate to early phases of development and later phases focus on execution and use of knowledge (e.g., production phases), it is not true to say that employees working in production do not create and develop new knowledge, because manufacturing processes can also be developed and improved based on employees' experiences.

Therefore in this study it is chosen not to categorizing individuals and their abilities to create, develop, share, and use knowledge under the concept of knowledge worker; instead, this paper focuses on different roles and how to support them because they are all special.

Employees contribute within one or more projects depending on their roles and responsibilities in the product development process. The project - which is a temporary organization with a fixed time and budget — has a specific task to solve, that is, a limited context to consider. There are tools that support management of knowledge with the purpose of storing and disseminating final results of a project, for example, in Knowledge Based Engineering (KBE), Product Data Management (PDM), and Product Life-cycle Management (PLM) systems. These are unlike experiences related to methods and the work process, which might be useful in other contexts and future projects as well, these systems have ben referred to as "heavy weight tools" (Bertoni et al. 2012). The perspective of "heavy weight tools" and rationale of the first wave of knowledge management (Huysman, and De Wit 2004) is not addressing the knowledge conversation as of this study. 
Nevertheless, tools, methods, and guidelines to support knowledge sharing do not consider how different roles form the messages in a knowledge conversation.

Typically, contributions to different knowledge dimensions are a mix of properties and attributes, some of them recognised by respondents. Respondents distinguish knowledge contributions depending on orientation in terms of confidence or characteristic contributionmeaning that a characteristic contribution relates to attributes that aim to support the customer value in focus, which may be, for example, safety or environmental care. One respondent explained that contributing to safety for the operator of a machine can mean installing a "dead-man's handle" or an auto-breaking circuit, but it may also mean improving the user manual, instructions, or visual warning system.

The confidence contribution tells more about the maturity of a technology area or project, and refers to the literature of Technology Readiness Levels (Mankins 1995) and Knowledge Maturity (Johansson 2009). Thereby knowledge conversations support an organization to define maturity of their knowledge base in different contexts and technology areas, but also to identify knowledge gaps and make decisions. The same respondent described this by using the metaphor of developing an engine. In this example, the goal of a project is to develop an engine for a new machine, and the company has a long tradition of making combustion engines. At first sight, it seems to be a relatively straightforward process, but new requirements put a fresh spin on this. The company needed new technology to meet the requirements; a hub motor or hybrid engine might be a preferred alternative. In such a case, experience developing combustion engines seems less relevant to bring up in knowledge conversations than increasing the confidence in the areas of hub motor and hybrid engine development.

Consequently, these two types of knowledge contribution address different audience, both in terms of group formations and roles of the individuals. Sveiby (2001) identifies three types of formations in knowledge transfer situations: namely, internal structure, external structure, and individual competencies. Sveiby (2001) then defines nine knowledge transfers on a strategic level, which show that transfer occurs differently in each transfer; for example, transfer from internal structure to external structure and transfer from external structure to internal structure are not necessarily addressed properly by asking the same questions.

This seems to be true as well as when knowledge conversations were analysed based on the relationship between the roles of the individuals who take part in a conversation. For instance, observations from conversations in a development project initiated by an engineer directed toward experts focused on feedback on the engineers' work activities. Once an engineer found out about a situation related to manufacturing quality, questions were asked in conversation with an expert, somewhat like: Have you experienced this before? Why did it happen? What could be the reason for this? What if I change this procedure like this? Could this be a preferred outcome/good result of the analysis?

That example relates to manufacturing of a transmission component; the same two individuals were later observed in a conversation again, now initiated by the expert aimed toward an engineer. At this time, the expert created new knowledge, interpreted, and defined it so that the engineer also could benefit from knowing it too. In this conversation, the expert possessed experience with a control system for the transmission that could be useful to the engineer; therefore questions should focus on how the expert developed and interpreted the situation, something along the lines of: What is the contribution? What are the benefits and/or challenges? What knowledge gap is addressed and how can this be used?

Observing the organization - which this paper intentionally aims to support-from a generic, high-level perspective, three roles were distinguished in the part of the company working with technology development: engineer, expert, and management. The management model from Kennedy and Sobek (2006) inspires these three roles, which reflect the main actors in early technology investigations and product development project. 
Management represents the role of employees working with activities related to the process of managing and controlling things or people. Management is responsible for ensuring that the team or organization progresses according to a plan.

Engineers possess knowledge and skill, which enables them to perform development activities. The capabilities of an engineer will be matched with the scope of a project and which resources are needed to fulfil its goal. A project manager defined this role as "the doers."

The expert can be described as a knowledgeable individual who possesses and is acknowledged for comprehensive knowledge in a specific context. The contribution of an expert is often related to a knowledge domain or a community in which he or she acts.

Managers tend to value technological interests over humans; despite it have prove to be a limitation for the development of technologies (Coggio 2013). Instead the management role can use the voice of an expert as a point of reference or clarifying a key concept when making decisions. An engineer will benefit from asking the expert for feedback on a specific task.

\section{Supporting Experience Sharing}

The relationship between the roles of engineers, management, and experts in knowledge conversations is explained through Figure 1. The arrows between the roles illustrate what kinds of messages are commonly discussed and asked for in these different knowledge conversations. It also reflects the four themes of questions developed based on the life-cycle perspective presented earlier. These questions were adapted to fit the purpose of each individual conversational direction of the nine that are addressed herein.

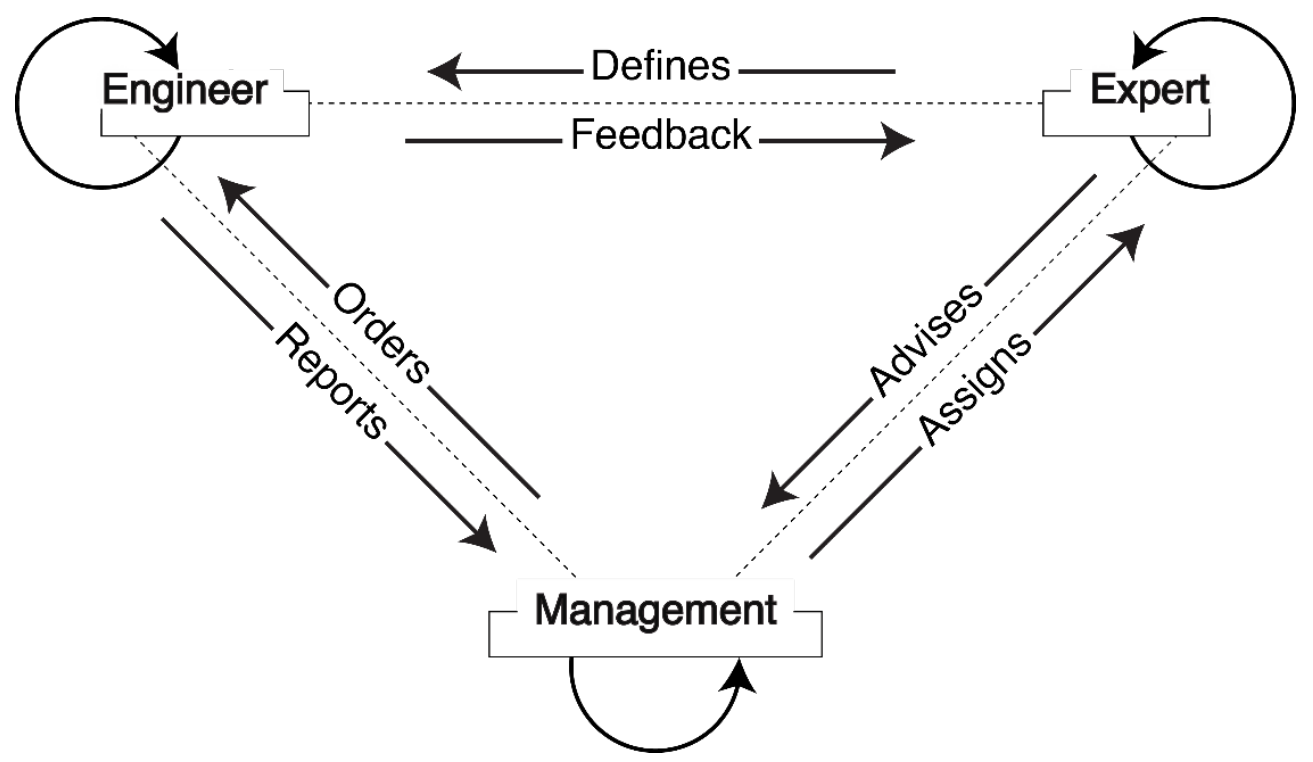

Figure 1. Knowledge conversation model (Source: adapted from Kennedy and Sobek 2006, 8)

Addressing specific roles (as shown in Figure 1) aims toward providing the right questions rather than the "right" answer to the wrong question. This implies identifying and sharing knowledge assets to gain contextual understanding. Some level of pre-knowledge is needed to enable the different roles to acquire knowledge from conversations with each other, which is also part of a reflective learning process.

One risk is making too general a statement and missing the rationale - that is oversimplifying a complex situation or making an effort to provide answers in a context that has not yet been in 
focus for a potential user of the message. The intention of such a message cannot be fully interpreted by a user in this case. Instead, knowledge conversations can support different roles in understanding what dimensions are necessary, can be directed toward the proper person and explained in what way experiences can benefit that role and also how that affects and makes a conversation fruitful for the involved roles. Moreover, because projects and knowledge projects in particular are assigned novel tasks, there are indications that preparing teams with a set of questions could help both capture and disseminate the experiences.

Support in terms of specific guiding questions, pointing toward the three roles-engineers, experts, and management - is outlined to reflect such a perspective. Therefore the questions are phrased so that all individuals can respond and contribute independently in the context in which he or she is working. These questions are all characteristic, depending on the roles that are involved in a knowledge conversation.

Existing support in the company encourages a knowledge-sharing culture, whereas how they share knowledge and to whom is often personally dependent, which is also supported by literature (c.f. Ghaznavi et al. 2013). Reporting on the final outcome of a project is more commonly directed to non-project member, meaning that the actual results of a development project are reported upon internally in reports, product specifications, presentations, and project seminars. Documentation of such work is stored in project portals with limited or no access to others besides project members. The project manager is responsible and assigned to that work, but the whole team is supposed to reflect upon their actions and address experiences from daily work so that others can learn from it. Knowledge and learning from individual project activities are missing in reports or seem to be treated as insignificant or trivial; they are not shared with employees other than those with whom they have to personally collaborate. This relates to the objective of an activity: that is, if the goal is to create an efficient product, then workers tend to manifest what has been achieved, instead of how they experienced their actions.

In existing support at the case company, templates for sharing experiences from projects provide questions and bullet lists of areas that encourage individuals to report technology outcomes in text, rather than to initiate conversations around their experiences. That is, an analysis made from observing projects onsite and reading documentation asking, "What went right/what went wrong? " is often interpreted, because one should talk about the performance of activities in relation to requirements. Reflections based on contributions and reflections related to existing knowledge are missing. If knowledge is acquired or missing, individuals as well as groups can benefit from reflection on experiences from doing an activity and sharing it with someone who also can find value from knowing the rationale behind results.

Therefore the following list of question is proposed as a result of this qualitative study, in a way and within a context that was not done before in literature. Addressing the question of how to make value out conversations in project teams and how to support different knowledge conversations and directing conversations to a specific role based on the model in figure 1:

- Management-Management

- What sets the context for your experience?

○ How could your experience contribute to organizational knowing?

$\circ \quad$ Why is the experience valuable for the organization?

$\circ$ How does your experience change existing knowledge?

- Management-Expert

- What knowledge domains does your experience address? 
- How can your experiences contribute to the mapping of available competencies?

- Why have you related your experiences to the chosen domain?

○ What proposal would be relevant based on your experiences?

- Management-Engineer

- Clarify what attributes and capabilities are lacking.

○ How will your experiences develop and/or match current knowing?

○ Why are your experiences important for engineering teams?

- What actions do you recommend based on your experiences?

- Expert-Management

- What are the critical competencies and knowledge for the organization?

○ How do your experiences relate to formalized knowledge?

- Why are your experiences an advantage for the organization?

○ How would you prioritize additional needed knowledge?

- $\quad$ Expert-Expert

○ What pre-knowledge supported your experiences?

○ How do your experiences create new knowledge?

- Why are your experiences and knowledge critical in this context?

- Suggest how your experiences could be fed into a new project.

- $\quad$ Expert-Engineer

-What is the contribution of your experiences to the engineering teams?

○ How did the challenge or possibility advance your experiences?

- What would you do differently if you were able to do it again?

○ Suggest how your experiences close knowledge gaps.

- Engineer-Management

- What is the relation between your experiences and organizational vision, goal, or purpose?

- How do your experiences contribute to coordination and matching of resources?

○ Why are your experiences useful for other situations in the organization? 
- What improvements or developments can you propose based on your experiences?

- Engineer-Expert

$\circ \quad$ What knowledge gaps you have identified?

○ How will your experiences support team competencies?

- Why is it crucial to identify this knowledge gap?

- What feedback and further actions are needed to make use of your experiences?

- Engineer-Engineer

-What pre-knowledge made you gain the experience?

○ How has your experiences affected your capability to conduct your task?

○ Why do your experiences support functions in a team?

○ Propose what your experiences provide for better execution of future teams' tasks?

\section{Conclusion}

Experiences are personal and dependent on specific conditions, but they are relevant for others to learn from. Therefore, the different roles of employees in an organization need to orchestrate their knowledge in conversations between one another to gain shared understanding. Hence, by possessing sound questions to reflect upon in knowledge conversations, it is reasonable to say that the contribution will be more mature in terms of general understanding within the topic.

Thus, decision-making and further development can be attributed to more than already known facts. Empirical data from interviewing representatives assigned to different roles in projects show that they interpret the existing support differently. It happens that they refer to the same situation but from different perspective and with different meanings. In such a case, it is necessary to provide support that can distinguish these differences, for instance, through the formulation of sound questions, which are listed in this paper.

The paper has proposed a structure for how to guide an organization in the process of identifying knowledge gaps and reflecting upon knowledge activities and, more precisely, for how to direct experiences to those who will benefit from knowing about them. Activities that today tend to be diminished in organizations can be supported by knowledge conversations.

Further, practitioners can benefit from this paper by understanding that not all benefit from the same guiding questions, and that they will not all give the same type of answers. The choice of guiding questions is carefully developed and matched with the objectives of different roles and their characteristic activities and tasks.

The perspective of global teams has not been the focus of this study and may be considered for future work. Personal relationships also seem to have great impact on knowledge conversations and need to be addressed in future studies. 


\section{Acknowledgements}

The author would like to thank the case company and the persons from the company for allowing open access to empirical data. The author is grateful to Åsa Ericson for supervision and comments on the manuscript.

\section{REFERENCES}

Argote, Linda, and Paul Ingram. 2000. "Knowledge Transfer: A Basis for Competitive Advantage in Firms." Organizational Behavior and Human Decision Processes 82: 150-169.

Bertoni, Marco, Andreas Larsson, Åsa Ericson, Koteshwar Chirumalla, Tobias Larsson, Ola Isaksson, and Dave Randall. 2012. "The Rise of Social Product Development." International Journal of Networking and Virtual Organisations 11(2): 188-207.

Blessing, Lucienne, and Ken Wallace. 1998. "Supporting the Knowledge Life-Cycle." In Proceedings of IFIP TC5 WG5.2 Third Workshop on Knowledge Intensive CAD 21-38.

Cheong, R Ricky K.F., and Eric Tsui. 2010. "The Roles and Values of Personal Knowledge Management: an Exploratory Study." VINE Journal of Information and Knowledge Management System 40: 204-227.

Coggio, Grace L. 2013. "Navigating Change during Innovation Diffusion: The Role of Management, Culture, and Communication in Technology Adoption." Change Management: An International Journal 12(4): 19-29

Davenport, Thomas H., and Laurence Prusak. 1998. Working Knowledge: How Organizations Manage What They Know. Boston: Harvard Business Press.

Disterer, Georg. 2002. "Management of Project Knowledge and Experiences." Journal of Knowledge Management 6: 512-520.

Fontana, Andrea, and James H. Frey. 2000. “The Interview: From Structured Questions to Negotiated Text." In Handbook of Qualitative Research, 2nd ed., edited by Norman K. Denzin and Yvonna S. Lincoln, 645-672. Thousand Oaks: Sage.

Ghaznavi, Mahmood U.O.K., Martin Perry, Paul Toulson, and Keri Logan. 2013. "Potential Enablers of Knowledge Collaboration in Ego-centered Networks of Professionals: Transactive Memory, Trust, and Reciprocity." Knowledge Management: An International Journal 12(1): 71-82.

Huysman, Marleen, and Dirk De Wit. 2004. "Practices of Managing Knowledge Sharing: Towards a Second Wave of Knowledge Management." Knowledge and Process Management 11(2): 81-92.

Ipe, Minu. 2003. "Knowledge Sharing in Organizations: A Conceptual Framework." Human Resource Development Review 2: 337-359.

Johansson, Christian. 2009. Knowledge Maturity as Decision Support in Stage-Gate Product Development. $\mathrm{PhD}$ Thesis, Luleå: Luleå University of Technology.

Joyner, Fredricka and Matt Wills. 2014. "Co-creating the Work Structures and Processes of the Future: The Interplay of Virtual Teams and Knowledge Management" Knowledge Management: An International Journal 13(2): 1-11.

Kennedy, Michael N., and Durward K. Sobek. 2006. "LAMDA: A Leadership Principle for Lean Product Development." In LAI (The Lean Advancement Initiative) Plenary Conference. MIT-Lean. San Antonio, Texas.

Mankins, John C. 1995. Technology Readiness Levels. White Paper.

Nonaka, Ikujiro. 1994. “A Dynamic Theory of Organizational Knowledge Creation.” Organization Science 5:14-37. 
Nuzzo, Paul, and Freyja Lockwood. 2005. Knowledge Enabled Solution Components: State of the Art. VIVACE Project Report.

Nonaka, Ikujiro. 1991. “The Knowledge-Creating Company.” Harvard Business Review 1-10.

Polanyi, Michael. 1966a. The Tacit Dimension. London: Routledge and Kegan Paul.

Polanyi, Michael. 1966b. "The Logic Of Tacit Inference." Philosophy 41:1-18.

QSR International, 2012. NVivo 10 Research Software for Analysis and Insight. Accessed November 24, 2012. www.qsrinternational.com/products_nvivo.aspx.

Rowley, Jennifer. 2001. "Knowledge Management in Pursuit of Learning: The Learning with Knowledge Cycle.” Journal of Information Science 27: 227-237.

Schmitt, Ulrich. 2015. "Towards a World Heritage of Memes Repository for Tracing Ideas, Tailoring Knowledge Assets and Tackling Opportunity Divides: Supporting a Novel Personal Knowledge Management Concept.” The International Journal of Technology, Knowledge and Society: Annual Review 10: 25-44.

Schön, Donald A. 1983. The Reflective Practitioner: How Professionals Think in Action. New York: Basic Books.

Sveiby, Karl-Erik. 2001. "A Knowledge-based Theory of the Firm to Guide in Strategy Formulation." Journal of Intellectual Capital 2: 344-358.

Volpentesta, Antonio P., and Alberto M. Felicetti. 2013. "Representing and Mapping Research Competencies within a Scientific Community" The International Journal of Knowledge, Culture, and Change in Organizations: Annual Review 12: 85-100

Webber, Alan M. 1993. "What's so New about the New Economy?" Harvard Business Review 71: 24-24.

\section{ABOUT THE AUTHOR}

Dr. Johan Holmqvist: PhD, Product Innovation, Luleå University of Technology, Norrbotten, Sweden 


\section{Management Education: An International Journal is}

one of four thematically focused journals in the

collection of journals that support The Organization

knowledge community - its journals, book series,

conference and online community.

The journal explores the dimensions of learning to lead in organizations that manage their knowledge resources effectively, have developed highly productive cultures and negotiate change effectively.

As well as papers of a traditional scholarly type, this journal invites case studies that take the form of presentations of management practice-including documentation of organizational practices and exegeses analyzing the effects of those practices.

Management Education: An International Journal is a peer-reviewed scholarly journal.

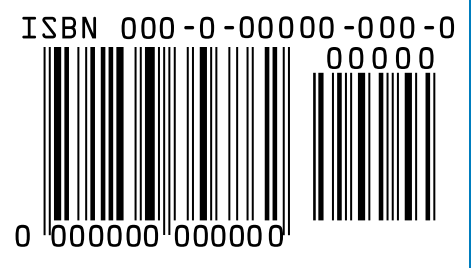

